\title{
Interplays between Mesoscopic Josephson Junctions and the Harper Equation
}

\author{
E. PAPP ${ }^{a, *}$, C. $\mathrm{MICU}^{b}$ AND I. BICA ${ }^{c}$ \\ ${ }^{a}$ Department of Theoretical Physics, West University of Timisoara \\ Bul. V. Parvan 4, RO-300223 Timisoara, Romania \\ ${ }^{b}$ Department of Physics, North University of Baia Mare, Str. Victoriei 76, RO-430122 Baia Mare, Romania \\ ${ }^{c}$ Department of Physics, West University of Timisoara, Bul. V. Parvan no. 4, RO-300223 Timisoara, Romania \\ Proofs are given that by resorting to the discretization of the superconducting phase variable leads to the \\ conversion of the eigenvalue equation of a mesoscopic Josephson junction under a dc voltage into a generalized \\ version of the Harper equation with anisotropy parameter. A full conversion proceeds, however, in terms of \\ selected parameters. Classical limits and further generalizations are also shortly discussed.
}

PACS numbers: 73.21.Cd, 74.50.+r, 74.78.Na

\section{Introduction}

The celebrated second-order discrete Harper equation $[1,2]$ :

$$
\varphi_{n+1}+\varphi_{n-1}+2 \Delta_{\mathrm{H}} \cos \left(2 \pi \beta_{0} n+\theta_{2}\right) \varphi_{n}=E_{\mathrm{H}} \varphi_{n}, \quad(1)
$$
where $n$ denotes an integer, serves to the description of the Bloch electrons on a two-dimensional lattice with nearest neighbor hoppings under the influence of a transversal and homogeneous magnetic field $B$. The number of magnetic flux quanta per unit cell is given by $\beta_{0}=B a^{2} e / h c$, where $a$ stands for the lattice spacing. The anisotropy parameter $\Delta_{\mathrm{H}}$ discriminates between metallic $\left(0<\Delta_{\mathrm{H}}<1\right)$ and insulator $\left(\Delta_{\mathrm{H}}>1\right)$ phases [3], while $\vartheta_{2}$ is a Brillouin phase. The energy $E_{\mathrm{H}}$, which is a periodic function of $\beta_{0}$ with unit period, is characterized by non-trivial butterfly-like nested band structures [2]. We have also to remark that the continuous limit of (1) is given by the Mathieu equation.

Equation (1), which is far from being trivial, exhibits rich structures and unexpected implementations in several areas. In particular, (1) has been applied to the description of superconductivity originating from linearized Ginzburg-Landau equations [4], of $d$-wave superconductivity with a magnetic field [5] and last but not at least to the quantum Hall effect [6] and Aharonov-Bohm cages [7]. We then have to realize that a deeper understanding of (1) is still in order. This motivates us to look in some more detail for interplays between mesoscopic Josephson junctions (MJJ) [8] and the Harper equation just mentioned above. For this purpose a generalized realization of (1) will be established in terms of a dis-

\footnotetext{
* corresponding author; e-mail: erhardt_papp_2005@yahoo.com
}

cretized version of the quantum-mechanical description of an MJJ under a dc voltage [9, 10]. Our main idea is that resorting to discretizations of variables provides a deeper understanding of phenomena [11].

\section{Josephson junctions under a dc voltage}

Circular superconducting rings of inductance $L$ with an MJJ under the influence of a dc voltage have received much interest $[9,10]$. The junction being mesoscopic means that the coherence length is larger than the dimension of the device, which opens the way to the onset of quantum interference phenomena. The influence of classical $[12,13]$ and quantized $[9,14,15]$ electromagnetic fields has also been discussed.

The basic Hamiltonian describing the superconducting ring with an MJJ reads

$$
H_{0}=-\frac{E_{\mathrm{C}}}{2}\left(\partial_{\theta}+\mathrm{i} \frac{Q}{2 e}\right)^{2}+E_{\mathrm{J}}(1-\cos \theta),
$$

where $\theta$ denotes the phase difference across the junction. The Coulomb energy is $E_{\mathrm{C}}=(2 e)^{2} / C$, while the energy $E_{\mathrm{J}}=\hbar I_{\mathrm{cr}} / 2 e$ characterizes the MJJ. The charge of the Cooper pair is $q_{\mathrm{C}}=-2 e<0$, as usual. Next, $C$ stands for the small capacitance of the junction, while $I_{\text {cr }}$ denotes the critical superconducting current. Switching on the dc voltage produces the dc charge $Q=Q_{0}=C V_{0}$. Our next step is to resort effectively to a one-dimensional ring description of radius $R_{0}$. We then have to account for a vector potential for which the azimuthal component reads

$$
A_{\varphi}=\frac{\hbar c}{R_{0}} \frac{Q}{(2 e)^{2}}
$$

which proceeds in cylindrical coordinates via $\varphi=\theta$. This 
gives the magnetic flux $\Phi=\oint A_{\varphi} \mathrm{d} \varphi$, where $0 \leq \varphi \leq 2 \pi$, as usual. The related number of flux quanta is then given by $\beta=\Phi / \tilde{\Phi}_{0}=Q / 2 e$, where now the flux quantum is $\tilde{\Phi}_{0}=h c / 2 e$. Accordingly, the number of the Cooper pairs exhibited by $Q$ is the same as the number of flux quanta, which reflects typical attributes of the superconducting "cooperon" description. We have to remark that the time independence of the above MJJ Hamiltonian complies from the very beginning with the time-independence of the Harper equation.

We would like to say that the charge induced under the influence of an external periodic flux

$$
\Phi_{\text {ext }}(t)=\Phi_{0} \cos \left(\omega_{0} t\right)
$$

is given by

$$
Q=Q_{\text {in }}(t)=-\frac{C}{c} \frac{\mathrm{d}}{\mathrm{d} t} \Phi_{\text {ext }}(t)=\frac{\omega_{0} \Phi_{0} C}{c} \sin \left(\omega_{0} t\right),
$$

where $\omega_{0}$ stands for the driving frequency.

Next we have to realize that the term i $Q / 2 e$ in $(2)$ can be ruled out in terms of the gauge transformation

$$
\psi(\theta)=\varphi(\theta) \exp \left(-\mathrm{i} \frac{Q}{2 e} \theta\right),
$$

in which case

$$
H_{0} \psi=\exp \left(-\mathrm{i} \frac{Q}{2 e}\right) \tilde{H}_{0} \varphi
$$

where

$$
\tilde{H}_{0}=-\frac{E_{\mathrm{C}}}{2} \partial_{\theta}^{2}+E_{\mathrm{J}}(1-\cos \theta)
$$

serves as a starting point for subsequent discretization.

\section{Performing the phase discretization}

Now we are in a position to perform the phase discretization $\theta=n \theta_{0}+\varepsilon$, where $\theta_{0}$ plays the role of a $\theta$-scale which remains to be established later. The additional $\varepsilon$ term has been inserted for convenience. On the other hand, one has the realization $\theta=2 e \Phi / h c$ [16], which also means that the corresponding flux discretization proceeds as $\Phi \rightarrow \Phi_{n}=\left(n \theta_{0}+\varepsilon\right) \tilde{\Phi}_{0}$. Conversely, starting from an integer flux discretization like $\Phi=n h c / 2 e$, yields the particular realization $\theta=n$ in accord with [16], where now $\theta_{0}=1$ and $\varepsilon=0$. The next step is to replace the second derivative $\partial_{\theta}^{2}$ in (8) by a product of discrete derivatives like [17]:

$$
\partial_{\theta}^{2} \rightarrow \frac{1}{\theta_{0}^{2}} \Delta \nabla
$$

which exhibits, of course, the continuous limit $\Delta \nabla \rightarrow$ $\theta_{0}^{2} \partial_{\theta}^{2}$. One has $\Delta f(n)=f(n+1)-f(n)$, which stands for the discrete right hand derivative. The left hand derivative reads

$$
\nabla f(n)=f(n)-f(n-1)=\Delta f(n-1) .
$$

It is clear that $\Delta^{+}=-\nabla$ and

$$
\Delta \nabla=\nabla \Delta=\Delta-\nabla \text {, }
$$

which shows that $\Delta \nabla$ is Hermitian. A deformed commutation relation like

$$
\Delta \Phi_{n}-\Phi_{n+1} \Delta=\theta_{0} \tilde{\Phi}_{0},
$$

should also be mentioned, which relies on quantum group generalizations discussed in some detail within the last three decades $[18,19]$. In other words, we found that the discretized counterpart of (8) is given by

$$
H_{\mathrm{d}}=-\frac{E_{\mathrm{C}}}{2 \theta_{0}^{2}} \Delta \nabla+E_{\mathrm{J}}\left[1-\cos \left(n \theta_{0}+\varepsilon\right)\right],
$$

which provides the eigenvalue equation

$$
H_{\mathrm{d}} \varphi_{n}=E_{\mathrm{d}} \varphi_{n},
$$

where $\varphi_{n}=\varphi\left(n \theta_{0}+\varepsilon\right)$ and $E_{\mathrm{d}}=E_{\mathrm{d}}\left(\theta_{0}\right)$. Looking for periodic solutions, say

$$
\psi(\theta+2 \pi)=\psi(\theta),
$$

leads to

$$
\varphi(\theta+2 \pi)=\exp (\mathrm{i} 2 \pi \beta) \varphi(\theta)
$$

where now $\beta=Q / 2 e$, which reproduces the well known Aharonov-Bohm twisted boundary condition.

\section{The onset of the generalized Harper equation}

Equation (14) can be rewritten equivalently as

$$
\varphi_{n+1}+\varphi_{n-1}+2 \tilde{\Delta}_{\mathrm{an}} \cos \left(n \theta_{0}+\varepsilon\right) \varphi_{n}=\tilde{E}_{\mathrm{d}} \varphi_{n},
$$

where

$$
\tilde{E}_{\mathrm{d}}=2\left[1+\frac{\theta_{0}^{2}}{E_{\mathrm{C}}}\left(E_{\mathrm{J}}-E_{\mathrm{d}}\right)\right]
$$

denotes the dimensionless rescaled energy, whereas

$$
\tilde{\Delta}_{\mathrm{an}}=2 \theta_{0}^{2} \frac{E_{\mathrm{J}}}{E_{\mathrm{C}}}
$$

has the meaning of a pertinent anisotropy parameter.

After having been arrived at this stage, let us perform a selection of the $\theta_{0}$-parameter via $\tilde{\Delta}_{\text {an }}=\Delta_{\mathrm{H}}$, which means in turn that $\theta_{0}$ gets established as

$$
\theta_{0}=\vartheta=\sqrt{\frac{\Delta_{\mathrm{H}} E_{\mathrm{C}}}{2 E_{\mathrm{J}}}} .
$$

The point is that under (20), Eq. (17) reproduces the Harper equation (1) when $\theta_{2}=\varepsilon$, but now in terms of the more general parameter $\vartheta$ instead of $\beta_{0}$. Accounting for (20) and proceeding further one finds

$$
\tilde{E}_{\mathrm{d}}=2\left(1+\Delta_{\mathrm{H}} \frac{E_{\mathrm{J}}-\bar{E}_{\mathrm{d}}}{2 E_{\mathrm{J}}}\right),
$$

where $\bar{E}_{\mathrm{d}}=E_{\mathrm{d}}(\vartheta)$, which becomes

$$
\tilde{E}_{\mathrm{d}}=3-\frac{\bar{E}_{\mathrm{d}}}{E_{\mathrm{J}}}
$$

as soon as $\Delta_{\mathrm{H}}=1$.

However, a parameter selection can be done such that

$$
\vartheta=2 \pi \beta=\pi \frac{Q}{e},
$$

where $Q=C V_{0}$, which corresponds reasonably to $2 \pi \beta_{0}$. This amounts to perform a dc-voltage tuning until

$$
V_{0}=\tilde{V}_{0}=\frac{2 \mathrm{e}^{2}}{C} \sqrt{\frac{\Delta_{\mathrm{H}}}{2 C E_{\mathrm{J}}}},
$$

when (17) becomes actually equivalent to (1), which reflects the parameter selection needed. We can then say that tuning the dc voltage in accord with (24) brings (17) into the Harper regime. Beyond (24), Eq. (17) is still a Harper-like equation, but with a rather different commensurability parameter such as done by (20). 


\section{Classical limits}

Choosing $Q=0$, one sees that (2) provides a pendulum with the effective mass

$$
m_{0}=\frac{\hbar^{2}}{2 E_{\mathrm{C}} R_{0}^{2}},
$$

under the influence of the rescaled gravitational acceleration $g=E_{\mathrm{J}} / m_{0} R_{0}$. The corresponding classical equation of motion is given by

$$
\ddot{\theta}+A \sin \theta=0,
$$

where $A=g / R_{0}$. Including damping effects and an $\mathrm{rf}$ driving of frequency $\omega_{0}$ produces the modified equation

$$
\ddot{\theta}+\alpha \dot{\theta}+A \sin \theta=B \sin \left(\omega_{0} t\right),
$$

instead of (26), where $\alpha$ denotes the damping coefficient and where $B$ stands for the strength of the driving force. It is understood that this latter term complies with (5). The interesting point is that (27) describes a driven MJJ [20-22], which opens the way to perform stability studies in terms of the asymptotic theory of nonlinear oscillations [23-25]. Discrete counterparts of the quantum-mechanical equations corresponding to (27) look promising for further studies.

\section{Conclusions}

In this paper proofs have been given that the quantum-mechanical eigenvalue equation characterizing an MJJ under dc voltage can be converted into a Harper equation with anisotropy parameter. For this purpose one resorts to the discretization of the phase difference across the junction, which opens the way to the introduction of right- and left-hand derivatives. Such conversions are also of interest for optical lattices [26]. In order to achieve a complete equivalence a suitable tuning of the dc voltage is in order, as indicated by (24). Besides a deeper understanding, we are by now in a position to establish useful relationships between MJJ's and several application areas of the Harper equation, like the quantum Hall effect or localization problems.

It should be stressed, however, that pulsating time dependent charges like (5) are able to be handled by resorting to the Floquet quasi-energy description [27, 28]. This results, however, in tight binding descriptions going beyond the capabilities of the Harper equation. Nevertheless, we can look for further generalizations of the Harper equation which should be able to account for (5) in terms of suitable time discretization schemes [29]. The incorporation of the spin-orbit coupling [30], of coherence effects [12, 31], as well as of strong couplings to quantized microwave fields [32], deserve further attention, too.

\section{Acknowledgments}

We are indebted to CNCSIS Bucharest and to the West University of Timisoara for financial support, as well as to H. Kleinert and M. Kibler for useful discussions.

\section{References}

[1] M. Wilkinson, Proc. R. Soc. Lond. A 403, 135 (1986).

[2] D.R. Hofstadter, Phys. Rev. B 14, 2239 (1976).

[3] S. Aubry, G. Andre, Ann. Isr. Phys. Soc. 3, 133 (1980).

[4] Y.Y. Wang, B. Pannetier, R. Rammal, J. Phys. (France) 48, 2067 (1987)

[5] Y. Morita, Y. Hatsugai, Phys. Rev. Lett. 86, 151 (2001).

[6] D.J. Thouless, B. Kohmoto, M.P. Nightingale, M. den Nijs, Phys. Rev. Lett. 49, 405 (1982).

[7] J. Vidal, P. Butaud, B. Doucot, R. Mosseri, Phys. Rev. B 64, 155306 (2001).

[8] K.K. Likharev, Dynamics of Josephson Junctions and Circuits, Gordon and Breach, Amsterdam 1996.

[9] A. Vourdas, Phys. Rev. B 49, 12040 (1994).

[10] Z. Jian, S. Bin, X.X. San, Phys. Lett. A 231, 123 (1997).

[11] E. Papp, C. Micu, Low Dimensional Nanoscale Systems on Discrete Spaces, World Sci., Singapore 2007.

[12] G. Schön, A.D. Zaikin, Phys. Rep. 198, 237 (1990).

[13] B. Shao, J. Zou, Q. Li, Phys. Rev. B 60, 9714 (1999).

[14] B. Shao, J. Zou, R. Wang, Phys. Lett. A 242, 105 (1998).

[15] L.M. Kuang, Y. Wang, M.L. Ge, Phys. Rev. B 53 , 11764 (1996).

[16] A. Vourdas, T.P. Spiller, Z. Phys. B 102, 43 (1997).

[17] A.F. Nikiforov, S.K. Suslov, V.B. Uvarov, Classical Orthogonal Polynomials of a Discrete Variable, Springer, Berlin 1991.

[18] L.D. Faddeev, N. Reshetikhin, L.A. Takhtajan, Leningrad Math. J. 1, 193 (1990).

[19] A. Connes, Noncommutative Geometry, Academic Press, New York 1994.

[20] R.L. Kautz, J. Appl. Phys. 62, 198 (1987).

[21] D.H. Dunlap, V. Kovanis, R.V. Duncan, J. Simmons, Phys. Rev. B 48, 7975 (1993).

[22] H. Seifert, Phys. Lett. A 101, 230 (1984).

[23] N.N. Bogoliubov, Y.A. Mitropolsky, Asymptotic Methods of Nonlinear Oscillations, Gordon and Breach, New York 1961.

[24] K. Tomita, Phys. Rep. 86, 113 (1982).

[25] E. Papp, C. Micu, Phys. Rev. E 64, 056203 (2001).

[26] Q. Niu, X.G. Zhao, G.A. Georgakis, M.G. Raizen, Phys. Rev. Lett. 76, 4504 (1996).

[27] X.G. Zhao, R. Jahnke, Q. Niu, Phys. Lett. A 202, 297 (1995).

[28] A.Z. Zhang, P. Zhang, D. Suqing, X.G. Zhao, J. Liang, Phys. Lett. A 292, 275 (2002).

[29] E. Papp, Int. J. Mod. Phys. B 20, 2237 (2006).

[30] J. Wang, K.S. Chan, J. Phys. Condens. Matter 21, 2457 (2009).

[31] G.E. Draganescu, A. Messina, A. Napoli, J. Mod. Opt. 56, 508 (2009).

[32] R. Migliore, A. Messina, J. Opt. B, Quantum Semiclass. Opt. 6, S 136 (2004). 